\title{
THE DIENES (MUTUAL INHIBITION) TEST IN THE INVESTIGATION OF PROTEUS INFECTIONS
}

\author{
M. B. SKIRROW* \\ Department of Microbiology, Children's Hospital, Birmingham \\ Plate XXXIV
}

THE use of the Dienes phenomenon for the study of cross-infection with proteus is not new (Story, 1954), but its reliability has been questioned (Kippax, 1957; France and Markham, 1968). An outbreak of proteus infection among babies born in a Birmingham maternity unit gave an opportunity to try to assess the value of the method more fully.

Dienes $(1946,1947)$ observed a curious interference of growth when unlike strains of proteus were allowed to swarm towards each other on an agar plate. Where the spreading halo of one strain met that of a different strain, the junction was marked by a line or ditch, readily visible macroscopically, caused by local inhibition of growth (fig. 1). Examination of the affected area by phase-contrast microscopy revealed that in it the long filamentous bacterial forms characteristic of the swarming type of growth gave rise to large globular bodies. No such effects could be detected, however, when the spreading haloes were of the same strain. Observing the behaviour of one strain of proteus against another in this way constitutes the Dienes test or mutual inhibition test (Cunliffe and Krickler, 1955). Obviously, only swarming strains can be tested by this method.

\section{METHODS}

The Dienes test. Conventional blood agar plates were employed. The medium consisted of a basal layer of Oxoid no. 1 blood agar base and a superficial layer of heart-infusion agar containing 5-10 per cent. horse blood, 1 per cent. tryptone, 0.5 per cent. $\mathrm{NaCl}$ and 0.005 per cent. para-aminobenzoic acid. The final concentration of agar was 1.4 per cent. and the

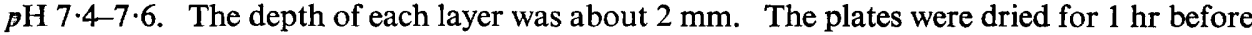
use and the organisms to be tested were spot-inoculated at opposite ends of each plate. Inocula were taken directly from surface cultures since a preliminary trial showed that results were the same as those obtained when inoculations were made from broth cultures. The plates were incubated at $37^{\circ} \mathrm{C}$ for $18 \mathrm{hr}$. Slow-swarming strains sometimes required a longer period of incubation, but if after $36 \mathrm{hr}$ their spreading growths had not met the test was repeated on a plate containing a thicker layer of agar, which increased their rate of spreading.

Multiple-test plates. Seven strains could be accommodated on one 9-cm plate in such a way that up to 12 combinations could be read at once (fig. 2). Occasionally an unusually fast- or slow-swarming strain made readings in this way impossible and the test had to be repeated. At first all tests done on the multiple-test plates were checked on single plates.

Received 8 Mar. 1969; accepted 3 Apr. 1969.

* Present address: Public Health Laboratory, Royal Infirmary, Worcester.

J. MED. MICROBIOL.-VOL. 2 (1969) 
The inhibitory reactions were always confirmed, but occasionally strains appearing compatible on a multiple-plate were found to be weakly inhibitory on a single plate. Consequently all compatible reactions on multiple-test plates were checked at least once on single plates; this did not outweigh the advantages of the former type of plate, since compatible reactions made up only 1.5 per cent. of the total.

Source of material. All swarming strains of proteus isolated from routine specimens (except faeces) were collected over a period of $7 \mathrm{mth}$. Isolates were numbered serially, except when the same strain-as judged by the Dienes test-appeared in the same patient more than once. Sometimes two or more distinct strains were found in a single specimen, in which case each was allocated a separate number. Thus a total of 300 isolates was collected from 279 patients. More than half of them were obtained from urine, but many of these were contaminants (table I).

TABLE I

Source of proteus strains

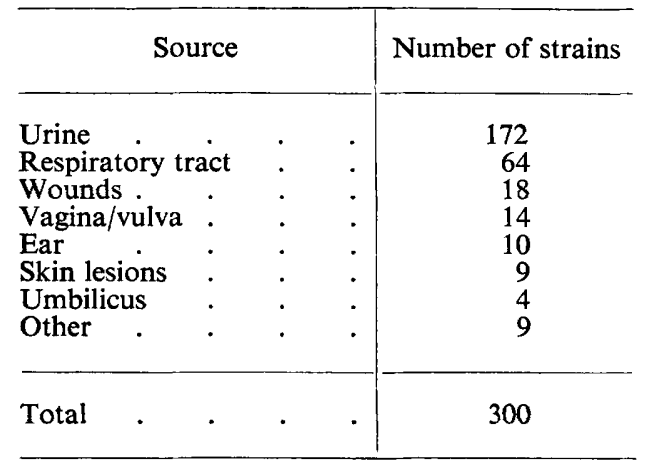

Cultures were maintained on nutrient agar slopes stored at room temperature. Biochemical tests were carried out according to the methods of Cowan and Steel (1965). Serological typing was performed by Dr B. Lányi of the State Institute of Hygiene, Budapest, to whom I am indebted for this service. The methods (Lányi, 1956) are based on the Kauffmann-Perch schema (Kauffmann and Perch, 1947; Perch, 1948).

\section{RESULTS}

\section{Observations on the performance and reading of the Dienes test}

Though the test is simple, unless one becomes familiar with the appearances of the plates it is easy to misinterpret the results. Blood agar cultures were found to be easier to read than those made on plain nutrient agar, and optimum viewing was by reflected light against a dark background. The surface of the agar had to be smooth and without blemish, since irregularities gave rise to confusing lines in the areas of swarming growth. The thickness of the medium had a marked effect on swarming: thick layers accelerated and thin layers retarded the spreading of a strain. Moist plates were difficult to read and adequate drying was therefore necessary.

The most likely source of error in reading the plates is the failure of one of the strains tested to swarm. In this event the swarming strain travels right across the plate to engulf the non-swarming colony and if the observer fails to notice this fact he will wrongly interpret the test as showing two compatible 
The Dienes TEST IN INFECTIONS

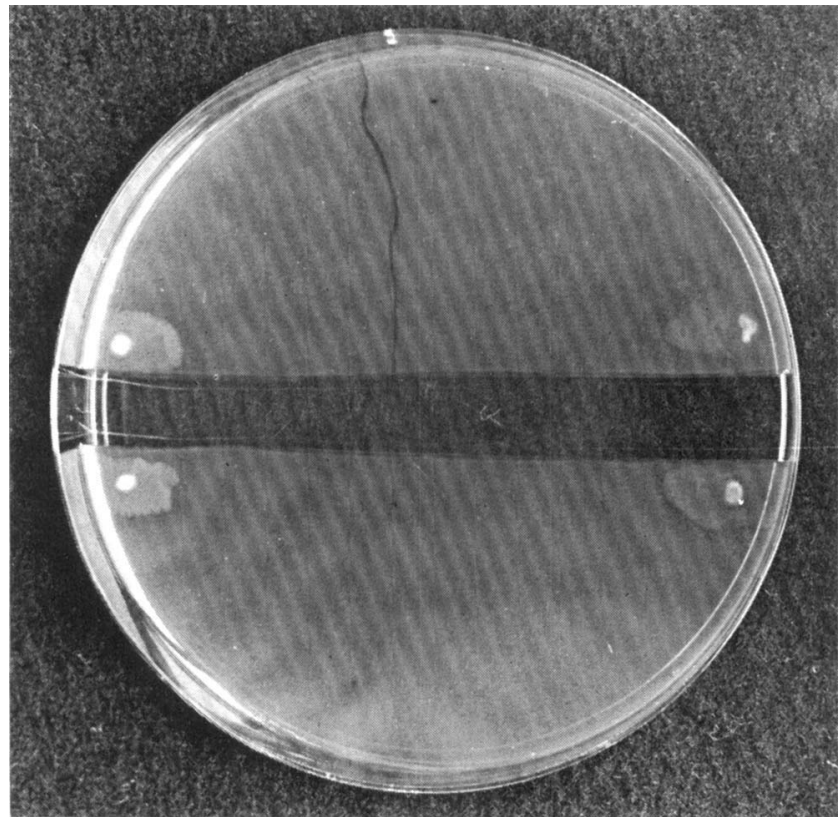

FIG. 1.-The Dienes test. An inhibitory reaction between 2 strains is shown on the upper half of the plate. On the lower half, one of the strains has been run against itself; there is no line of inhibited growth at the junction of the two swarming haloes, which merge imperceptibly into one another.

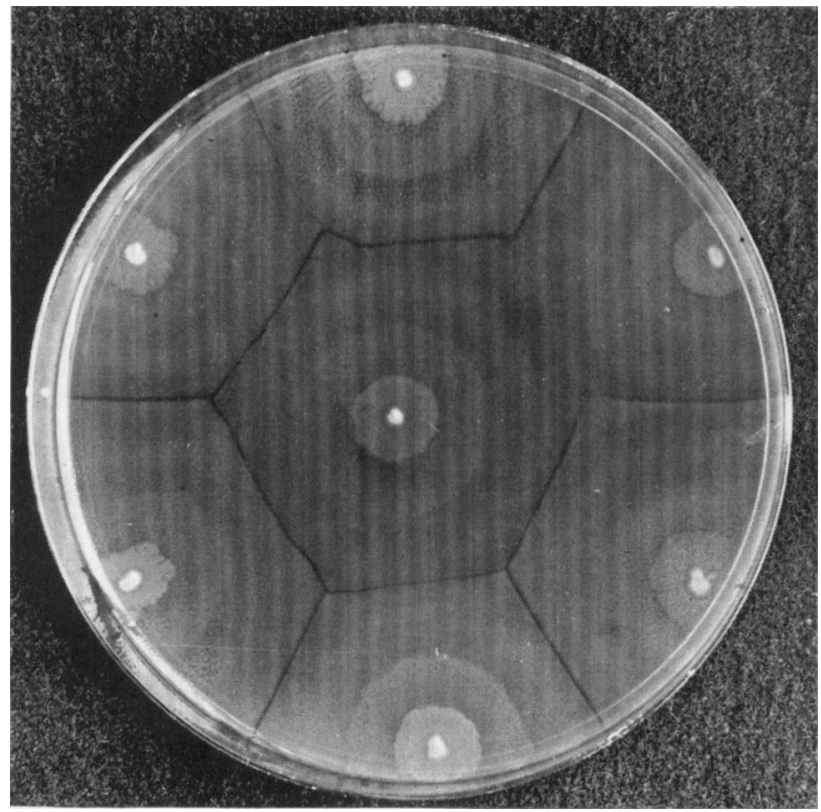

FIG. 2.- Seven strains have been inoculated on a single plate. The "epidemic strain" is in the centre; it is compatible with one of the other strains. The other 5 strains show the typical inhibitory reaction. 
strains, since no line of demarcation is visible. This error can be avoided by observing the concentric rings formed by the alternating stages of swarming and stationary growth characteristic of phase A (Belyavin, 1951); the origin of the swarming growth can be determined by noting on which colony these rings are centred (figs. 1 and 2). A form of swarming has been described that is continuous (Belyavin's phase C); if one or other of a pair of test strains is in this phase, according to Belyavin they appear compatible to the Dienes test. This type of growth was not encountered in the course of my experiments.

As Dienes originally observed, mutual inhibition does not occur if the swarming growth of one strain by chance meets the other when the latter is temporarily in a stage of stationary growth, i.e., when contact between the two strains coincides with the edge of one of the concentric rings. This is likely to be a source of error only with slow-swarming strains in which the concentric rings are close together. In such cases, the test has to be repeated, preferably on thicker agar which encourages the slow strain to travel faster.

Though the majority of tests were distinct and easy to read, there were a few in which there was a diffuse thinning of growth rather than a well-defined line where the two strains met. This effect was reproducible with the same pair of strains, and as some pairs were shown to be different in other respects as well, such weak reactions were taken to indicate genuine strain differences.

\section{The use of the Dienes test in the investigation of an outbreak of cross-infection with proteus}

The test proved to be of great value in the investigation of an outbreak of proteus infection among infants born in a Birmingham maternity unit. Three infants died from proteus meningo-encephalitis within a few days of each other. The first one came to necropsy at the Birmingham Children's Hospital and Proteus mirabilis was isolated from the umbilical artery, blood and brain. This strain will be referred to as the " epidemic" strain. Similar findings were obtained in the second infant whose necropsy was done at a different hospital, and the Pr. mirabilis which was isolated gave a compatible reaction in the Dienes test with the epidemic strain. Material was not obtained from the third baby in whom the diagnosis was made on clinical grounds.

Umbilical swabs were obtained from eight other infants who had been born in the maternity unit and were contemporaries of the dead babies. Pr. mirabilis was isolated from all eight. Seven of these isolates were compatible in the Dienes test with the epidemic strain and with each other; the remaining strain was inhibitory to the epidemic strain and to the other seven.

In the course of the next few months, the epidemic strain was tested against the 300 proteus isolates obtained from routine specimens. Compatibility in the Dienes test was found with only 5 isolates; the remaining 295 were inhibitory to the epidemic strain and were therefore judged to be distinct from it. The 5 compatible strains were also compatible with each other; it was subsequently shown, however, that they were not all of the same serological type. The epidemic strain was serologically distinct: O26:H3 (Lányi's modification of 
Kauffmann-Perch schema). Four of the 5 compatible strains were type $\mathrm{O} 26: \mathrm{H} 2$ and one was $\mathrm{O} 21: \mathrm{H} 2$. The rarity of the epidemic strain was further confirmed by Drs E. J. L. Lowbury and J. L. Whitby, who used both the Dienes test and serological typing methods in their search for it (personal communication). A compatible strain was found only once during the examination of hundreds of proteus strains from the affected hospitals and from other hospitals in the Birmingham area, but this too was later found to be serologically distinct. In the light of these findings, the compatibility in the Dienes test between the isolates from the maternity unit babies becomes highly significant. There can be little doubt that they were isolates of one and the same strain since they were also serologically identical.

Strains of proteus isolated from five other unrelated cases of infantile meningo-encephalitis and septicaemia encountered over a three-year period were found to be distinct from the epidemic strain and from one another by means of the Dienes test.

\section{Reactions among strains of proteus isolated from patients and staff of two children's wards}

Patients and staff of an infants' ward (consisting of a flat of cubicles each containing two cots) and a children's ward were screened for faecal carriage of proteus (table II). Although measures to prevent cross-infection were operative in the infants' ward, there was ample opportunity for cross-infection to take place in the children's ward, which was inhabited by active toddlers. The proteus isolates in each group were tested against each other by the Dienes method. Only in one instance was a compatible pair of isolates found; they were from two babies that had shared the same cubicle for several weeks. The high carrier rate among the infants was in accordance with previous unpublished observations made in our laboratory.

\section{Compatible reactions among strains isolated from unrelated sources}

In order to obtain a better estimate of the specificity of the Dienes test, the first 48 isolates of the series of 300 were tested against each other in every possible combination. This involved a total of 1128 tests of which only 16 showed compatibility between strains, i.e., an average of one compatibility in every 70 tests. This is a similar rate to that produced by the epidemic strain with the 300 isolates.

The 48 isolates can be grouped according to their compatibilities; in fact they fall into 35 groups or "Dienes strains". Twenty-seven of the 35 Dienes strains were represented by only one isolate, 4 strains by two isolates, 3 strains by three isolates and 1 strain by four isolates. Whether the isolates belonging to a single " Dienes strain " were also related serologically was not investigated.

\section{The relation of the Dienes test to different biotypes}

Each isolate was tested for the production of indole and fermentation of sucrose. An organism giving a negative reaction in both tests was classed as 
Pr. mirabilis. If one or other of the tests was positive, the organism was further tested for the fermentation of maltose. Indole-positive, maltose-positive organisms were classed as $P r$. vulgaris.

On this basis, of 294 strains tested, 285 proved to be Pr. mirabilis, 8 Pr. vulgaris and 1 was an indole-negative, maltose-positive variant (atypical type I of Kippax). Seven of the $8 \mathrm{Pr}$. vulgaris strains were compatible with one another, i.e., they were regarded as the same " Dienes strain ". Reactions between the Pr. vulgaris and Pr. mirabilis strains were often indistinct and of the weak type, although other differences in the growth characteristics, such as colour and production of haemolysis, were usually evident. These observations are in accordance with those of Kippax, who noted that different biochemical strains sometimes swarmed together; indeed this was one reason why he abandoned the Dienes test in favour of biochemical tests for strain differentiation.

TABLE II

Faecal carriage of proteus in two types of children's ward

\begin{tabular}{lr|c|c|c|c}
\hline \multicolumn{1}{c|}{ Type } & $\begin{array}{c}\text { Number of } \\
\text { infants tested }\end{array}$ & $\begin{array}{c}\text { Number of } \\
\text { proteus strains } \\
\text { isolated from } \\
\text { infants }\end{array}$ & $\begin{array}{c}\text { Number of } \\
\text { staff tested }\end{array}$ & $\begin{array}{c}\text { Number of } \\
\text { proteus strains } \\
\text { isolated from } \\
\text { staff }\end{array}$ \\
\hline Infants' ward (cubicled) & $\cdot$ & 18 & 13 & 37 & 2 \\
Children's ward (open) & $\cdot$ & 21 & 6 & 12 & 4 \\
\hline
\end{tabular}

The reproducibility and long-term stability of the Dienes test

Tests were frequently repeated during the early part of the work, but no significant variation was found: reactions that were inhibitory remained inhibitory and those that were compatible remained so.

The retesting of strains was then extended to cover a longer period. When proteus was isolated from a patient on more than one occasion, the most recent isolate was tested against the original one in the Dienes test; only those obtained more than $1 \mathrm{mth}$ apart were tested in this way. Most of these isolates came from children with diseases requiring long-term follow-up, such as chronic renal disorders and chronic chest infections associated with cystic fibrosis. Seventy-six such "pairs" were collected. Of these pairs only 9 were inhibitory to one another indicating a change of strain. The remaining 67 pairs were compatible and there is little doubt that each patient was continuing to harbour his or her own strain. The average interval between isolations was $6 \mathrm{mth}$. Three compatible pairs were obtained a little over $2 \mathrm{yr}$ apart; these organisms were cultured from the sputa of 3 children suffering from cystic fibrosis. One of these pairs is of particular interest, since resistance to ampicillin had developed between the time of the first isolation on 5 Sept. 1966 and the last on 26 Sept. 1968; the two isolates remained, however, compatible in the Dienes test. 


\section{Discussion}

The frequency of compatible reactions due to chance that can be expected to occur in the Dienes test between proteus isolated from " unrelated " sources is in the region of 1 in 70. This estimate is based on the reaction of the epidemic strain with 300 isolates obtained from routine hospital material and on the testing of 48 isolates against themselves. This degree of specificity makes the test valuable for the detection of cross-infection with proteus, as shown by my investigation of the outbreak in the maternity unit. There is no evidence for the predominance of any particular " Dienes strain ", judging by the results of the work on 48 isolates, 35 of which were the sole representatives of their strain. The largest number of isolates in any one "Dienes strain " was 4.

In its present form the only way the Dienes test can be used is to identify a strain by its compatibility with other strains. Whilst often this is all that is required in the investigation of cross-infection, a system of typing for the study of the general epidemiology of proteus infections would seem desirable. The results of the present experiments suggest that such a system based on the Dienes phenomenon might prove feasible.

The work of Cunliffe and Krickler (1955) suggested that the H-antigen is the chief determinant of compatibility in the Dienes test. They found that proteus strains possessing different $\mathrm{H}$-antigens were always inhibitory, even though their O-antigens may have been the same. They described one instance of compatibility in which the two strains shared a common $\mathrm{H}$-antigen, but had different $\mathrm{O}$-antigens. My results show that it is possible for strains to possess different $\mathrm{H}$-antigens and still be compatible in the Dienes test: the epidemic strain $\mathrm{O} 26: \mathrm{H} 3$ was compatible with serotypes $\mathrm{O} 26: \mathrm{H} 2$ and $\mathrm{O} 21: \mathrm{H} 2$. It is therefore clear that the behaviour of strains as regards the Dienes phenomenon is not governed solely by $\mathrm{H}$-antigenic structure. In any case, if this were so, one would have to postulate the existence of many more different $\mathrm{H}$-antigens than those hitherto described (but see Addendum).

It is difficult to imagine how flagellar differences could produce the changes described by Dienes. The formation of large bodies appeared to be related more to noxious influence such as refrigeration, solutions of low osmotic pressure and chemical substances (Dienes, 1946). Furthermore, Dienes observed that the haloes of unlike strains sometimes stopped at a short distance from each other without coming into actual contact, thus suggesting the diffusion of antagonistic substances into the medium. Hughes (1957) revealed something of the complexity of the situation by his studies on the swarming of proteus. His experiments indicated that swarming is induced by the action of toxic substances of at least two kinds: one is a non-specific volatile agent that is not detectable after growth has been cleared from the medium and exerts a greater effect when cultures are confined under a coverslip; the other is a strainspecific, stable, non-volatile agent (or agents) that remains in the medium after clearance of the primary culture. It is possible that these strain-specific substances induce swarming at low concentrations, but inhibition at higher concentrations, and that they are the agents responsible for the Dienes phenomenon. Such substances might perhaps be related to colicines. 


\section{SUMMARY}

The technique of performing the Dienes test is described, as well as its application to the investigation of an outbreak of cross-infection with Proteus mirabilis. The test was found to possess a high degree of specificity. The frequency of reactions of identity among 48 proteus isolates obtained from unrelated sources and tested against themselves was 1 in 70 , based on a total number of 1128 tests. These 48 isolates could be grouped into 35 " Dienes strains".

Compatible reactions were obtained between strains possessing major $\mathrm{H}$-antigenic differences. This finding indicates that the relation between $\mathrm{H}$ antigenic structure and inhibition in the Dienes test is probably not as close as has been previously suggested.

In a study of children attending hospital for up to $2 \mathrm{yr}$, strains of proteus repeatedly isolated from a child were almost always compatible with each other in the Dienes test. This indicates that each child retained his or her own strain and that the strains behaved consistently in relation to the Dienes test during the period of the study.

It is concluded that the specificity, reproducibility and simplicity of the Dienes test make it of great value for the detection of cross-infection with proteus, particularly in an ordinary hospital laboratory. The suggestion is made that the Dienes phenomenon could be due to colicine-like substances and that it could form the basis of a typing system.

I am indebted to Dr B. Lányi of the State Institute of Hygiene, Budapest, for the serological typing of six proteus strains. I wish to thank Dr K. B. Rogers and Dr E. J. L. Lowbury for their interest and advice generously given, and $\mathrm{Dr}$ R. J. Henderson for suggestions concerning the preparation of the manuscript.

\section{REFERENCES}

Belyavin, G. . . . . . . . . 1951. J. Gen. Microbiol., 5, 197.

Cowan, S. T., AND Steel, K. J. . . . 1965. Manual for the identification of medical

Cunliffe, A. C., and Krickler, Maureen 1955. Atti VI Congr. int. Microbiol. (Roma, S. 1953), 1, 861.

Dienes, L. . . . . . . . . . 1946. Proc. Soc. Exp. Biol. Med., 63, 265.

". . . . . . . . . . 1947. Ibid., 66, 97.

France, Diana R., and Markham, N. P. 1968. J. Clin. Path., 21, 97.

Hughes, W. H. . . . . . . . 1957. J. Gen. Microbiol., 17, 49.

Kauffmann, F., and Perch, Beate . . 1947. Acta path. microbiol. scand., 24, 135.

Kippax, P. W. . . . . . . . . 1957. J. Clin. Path., 10, 211 (August).

LÁNYI, B. . . . . . . . . . . . . 1956. Acta microbiol. hung., 3, 417.

Perch, BeAte . . . . . . . . 1948. Acta path. microbiol. scand, 25, 703.

StORY, P. . . . . . . . . . 1954. J. Path. Bact., 68, 55.

\section{Addendum}

Since this was written de Louvois (1969, J. Clin. Path., 22, 263) has shown that strains of proteus that are serologically identical may be of different Dienes types. 